\title{
Article \\ Experimental study of the forces and parameters for the opening of incisions inside ocular cavity
}

\author{
V. O. Ramírez-Beltrán ${ }^{1}$, L.A.Z.-Avilés ${ }^{1,2,3 *} \mathbb{C}$, R. M. Valdovinos-Rosas ${ }^{1}{ }^{\mathbb{C}}$, J. J. Reyes-Lagos ${ }^{2} \mathbb{C}$ \\ and G. M. Cruz-Martínez ${ }^{1}$ (D) \\ 1 Faculty of Engineering, Autonomous University of Mexico State, 50130, Toluca, México. \\ 2 Catedras CONACYT, Autonomous University of Mexico State, 50130, Toluca, México. \\ 3 Faculty of Medicine, Autonomous University of Mexico State, 50120, Toluca, México. \\ * Correspondence: lazunigaa@uaemex.mx; Tel.: +52 5512018024
}

\begin{abstract}
The experimental results of forces and efforts derived from the opening of incisions in the orbital cavity in a pig's head are presented in this article. The different areas of the incision openings are related to the needs at the incision procedure for a dacryocystorhinostomy. In terms of experimental procedure, an origin and a plane are defined so as to allow the location of the opening of the incision. The incisions are retracted along an axis of said origin. This procedure has been based on the mathematical model developed for this work, which consists of a procedure for determining the behavior of an incision when a force is applied to retract the skin. The experimental data obtained, suggests the existence of an almost linear relationship between the increment of resistance in relation to the time obtained for each opening, same of which is deemed to be consistent with the behavior of an elastic material.
\end{abstract}

Keywords: Incisions opening, Forces, parameters, ocular cavity

\section{Introduction}

According to the National Institute of Statistic and Geography of Mexico (INEGI), there are 5.700 million people with a disability and $27.2 \%$ are ocular disabilities in Mexico [1,2]. The Ophthalmology Mexican Society estimates that ocular disabilities are the most growing diseases in Mexico and the World Health Organization consider that $80 \%$ of ocular pathologies can be prevented of cure [3]. The most common surgical procedures in Mexico are lacrimal apparatus block-age, conjunctivitis, glaucoma, corneal abrasion (or corneal injury), stye, chalazion, cornea transplant pterygium transplant [4].

The eye, to prevent o minimize further damage, requires cares before, during and after a surgical procedure [4-6]. While surgery is performed, the small working spaces difficult the precision of the specialist. An example of this problem is the surgery called Dacryocystorhinostomy (DCR), which prevents the obstruction of the lacrimal apparatus allowing a normal drain directly into the nasal cavity [5].

In a DCR a tangential incision among $2-4 \mathrm{~cm}$ of length is retracted until the lacrimal sac is found $[5,7,8]$. The identification and exposure of tissues and skin is an important step during the DCR allowing better mobility and space needed for the surgeon [7].

Although lacrimal surgeries are considered safe, its location, between the ocular cavity and the nose, difficult the handling of the tools on the procedure. To perform the surgery on these small working spaces, the surgeon needs the help of medics and nurses with knowledge in DCR [5,7].

Medical tools for retraction do not consider the amorphous area of the incision, the ocular cavity and the nasal bone varies for each patient. The inhomogeneous surface and workspace area increase costs, time of surgery and decreases the possibilities of a successful procedure. 
This problem generates considerations in the process of incisions and the opening workspace stage. This process is divided into this article in the characteristics of the skin and the forces, lengths, deformations that the retraction could have.

The skin is the most extensive organ of the human body. This organ allows the interaction between the organs and tissues and the exterior of the body [9]. It has specific functions and properties that protect the whole body and allows to exchange substances with the environment $[9,10]$.

When an incision is made, it is necessary to comprehend several aspects of the surgery such as the anatomy of the incision zone, the desired depth of the incision and the structures or organs adjacent to the procedure [11]. Several types of incisions can be performed with a scalpel, and these vary from the type of injury to be treated [12]. The tangential incision is the most common incision that allows log in at small lesions and that do not have high depths [11].

Once the cut is made, it is necessary to retract the skin and tissues adjacent in the working area $[12,13]$. For this procedure, there are manual or mechanical separators used to release the interest area and be able to perform the surgery [13]. The use of these tools will depend on the type of surgery and the zone to perform the surgery [14].

There are mechanical retractors like Transoral robotic-assisted [13,15], Transoral retractor for robotic surgery [13] with rigid elements useful in operations with spaces above $10 \mathrm{~cm}$ and a semi plane incision [15]. These retractors are not suitable to use in DCR due to the adaptability and size. Ordinary Medical Instrumental for DCR (OMD) are tools for opening the incisions, steel retractor or cold scalpel, used in a DCR make mobility difficult and reduce the workspace for the specialist $[5,14]$. Hence, this work contribution is focused on specifying the design parameters of an adaptative device for this kind of surgery.

The manual tools require that the surgeon be assisted by specialists on ocular surgery in order to manipulate the retracted incision, causing difficulties of mobility and manipulation of tissues during the surgical procedure. In a DCR, the tools for opening the incisions used make mobility difficult and reduce the workspace for the specialist [5]. The manual tools require that the surgeon must be assisted by other specialists in the manipulation of the retracted incision, causing difficulties of mobility and manipulation of tissues during the surgical procedure [7].

This problem generates a need to perform a Robotic Retraction System (RRS), which retracts the incision automatically reducing problems of workability and workspace for the incision. Make a RRS is necessary to know the design parameters that the robot must have for optimal performance. The construction of the RRS was divided into specialist needs, obtaining a mathematical model that predicts the behaviour of the skin during the opening of the incisions and finally, the requirements of the RRS. The RRS design parameters are determined by the comparison of al elements and characteristics found on the methodology User Satisfaction Survey (USS), which is a qualitative methodology that compares the specialist needs, determined by semi-structured interviews, and the parameters of the system itself [16-18].

The proposed theoretical considerations are specific parameters in order to find out the minimal points to retract the incision in a homogeneous form, the characteristics are immersed on a Mohr tensor circle [19]. For the requirements, parts were determined according to the properties of the incisions on theoretical review and the surgeries observed $[16,20]$. The system requirements are the forces and efforts necessary to open and maintain a constant workspace during a procedure.

In the experimental collection of the retraction forces, a pig head was used as sample tissue [7,9]. The pig shares genomic similarities that allow the analysis of this animal as a model for biomedical research from the anatomical and physiological point of view, the pig has been used to heal humans in cardiac surgery with aortic valves, and production of heparin, an anticoagulant, and possible organ transplantation $[9,10,21]$.

The present work has as purpose determining the specifications of forces and lengths needed to open an incision during a DCR. Those specifications are part of the RRS design parameters on different types of opening areas. The results show a parametrical relation between forces applied in a point 
to retract the skin at two different lengths. The retraction study shows that the incision should be retracted at least 6 points in order to have a homogeneous workspace.

In the following sections are presented the determinations of the needs for the specialist, the obtaining of the requirements for the SRR and the experimental results obtained in the experiment of the opening of the incisions in the orbital cavity of a pig's head.

\section{Methodology}

The USS methodology is made to define the user needs on a specific problem [16]. Those needs define the system criteria's and allow to generate the parameters that the system could solve.

On the present work, the USS is divided into 3 stages, interview with a specialist to know the specialist needs on the DCR opening-retraction process; observe surgeries that provide the surgical criteria's and the documentation generating the technical criteria's for the process.

With the technical criteria's defined, the USS methodology continues the testing protocol in order to obtain the metrics of the opening retraction process. All the stages are shown in Figure 1 and developed in the following sections.

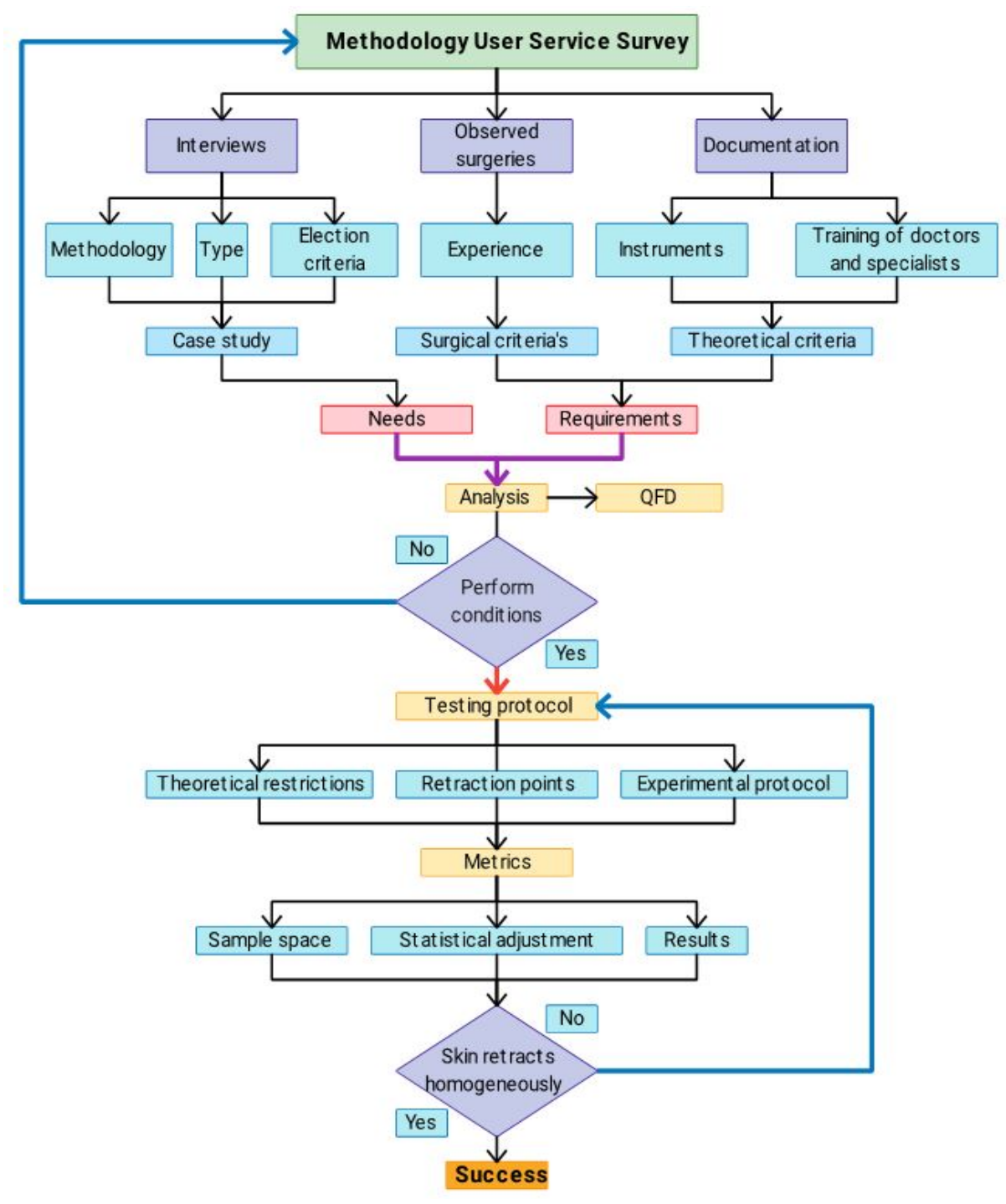

Figure 1. Methodology defined to design an RRS. 


\subsection{Interview with specialists}

Due to the DCR procedure is a technical and specific procedure, the information acquisition was made by qualitative interviews. This type of interview has been useful in research with topics in surgery by contributing technical information of the surgical process and its difficulties [16,18].

Within the qualitative interview, there is a subtype of inter-view that allows to go deeper on a specific topic and is called Semi-structured interviews [16]. Semi-structured interviews consist on to ask a series of open questions at the beginning of the interview and deepen the topic that the interviewer consider important.

The information collected result on the necessity of knowing the "forces, lengths and efforts resulting in the incision retraction process on a DCR".

\subsubsection{Case study.}

The case study can be defined in the opening points, the safety criteria and the operating principles for the whole system.Given the user needs are related on masses and length, the incision retraction process was replicated in death tissue of a pig's head that has similar mechanical characteristics of the human skin [9-12,21].

A DCR is performed in areas between the ocular cavity and the nasal bone and the replication was performed in the same area and with de same incision length $(2 \mathrm{~cm})$. The retraction process is a mechanical manipulation of the material which can be considered as a flexible material and with an approach, a retraction point could generate parametric results in different forces applied in an incision. This particularization of the problem should obtain the internal and external metrics for the retraction process.

\subsubsection{Surgical Needs.}

The interviews determined the surgical needs, listed below.

open on the desired area.

\& Retract a diverse area.

is Retract a diverse depth.

A safe on the retraction processes.

as No electric.

a No noisy.

is Economic.

4 Liberate the workspace.

4. Maintain the workspace on a specific period.

as Easy manipulation.

Those needs represent the principal difficulties that the surgeon faces on a DCR and are compared on a QFD (Figure 2) for its analysis.

\subsection{Surgeries observed.}

In the observation of DCR was possible to understand the difficulties externalized by specialists. When an incision needs more tools for its retraction, the time of surgery increases. The specialist retracts the incision on a homogeneous geometry and the most common figure is an ellipse. Whit the observation process was possible to define the following surgical criteria's.

\& Define several retraction points.

as Define several retraction depths.

is Retraction limitation.

क Simplify the retraction process.

a Reduce the staff in a DCR.

The Surgical criteria's are considered a part of the parameters needed to compare with the necessities on the QFD (figure 2). 


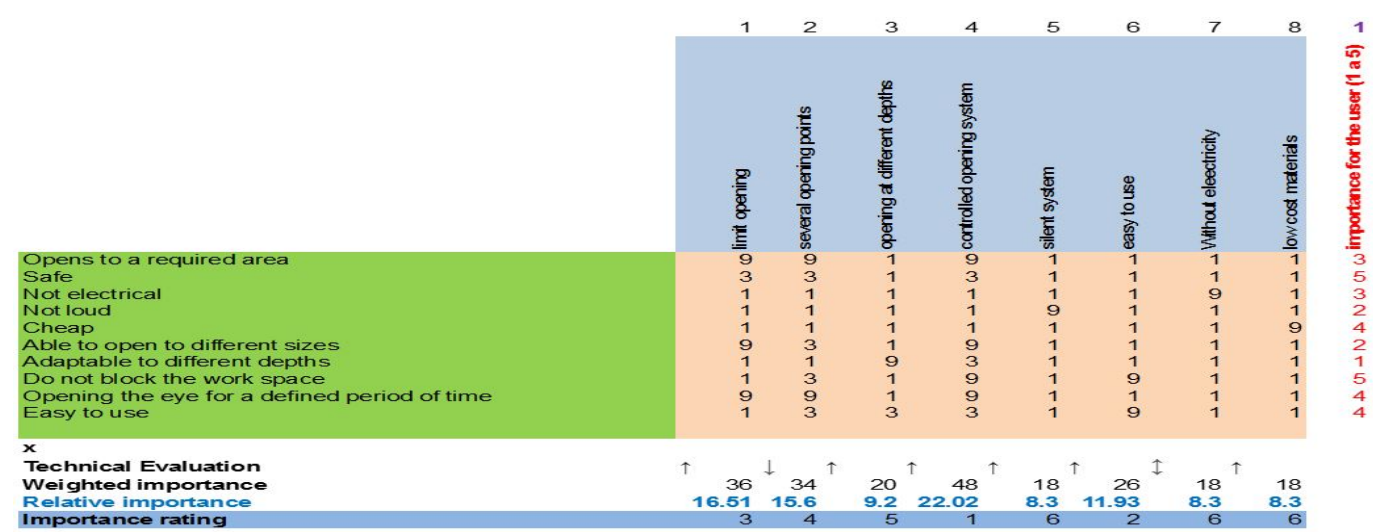

1. Must have an opening system controlled by the specialist and limitated by the pressure supported.

2. Must works with a system easy to handle by the
specialist

Specialist Must limit the opening considering the pressure.

4. Must establish opening points considering the required work space and the user safety
5.Must consider different opening depths appropriate to the needs of the specialist

5. Must consider different opening dept
6.Must be a silent operati ng system

7.Must work without electricity

8. Must be built with low cost materials and systems

Figure 2. Given the user necessities the parameters founded on the surgeries observed and the documentation stages, it was possible to realize the following House of Quality.

\subsection{Documentation.}

The anatomical definitions and clinical concepts are necessary to understand the pathologies. On this stage, it is been cata-loged the instruments needed in incision retraction process on a DCR.

Table 1. Instruments for an incision retraction in a DCR [6].

\begin{tabular}{clc}
\hline & Instruments & \\
\hline Anesthesia & Vaso-builder spray & Antiseptic \\
Cold scalpel & Scissors & Retractors \\
Guide drill & Hemostatic & Kerrison clamp \\
& mosquito clamp & \\
Curved mosquito & Wescott scissors & Silicone tubes \\
\hline
\end{tabular}

Those instruments are handled manually by under training ocular surgeons, process identify during the surgical observation stage, and the number of specialists could change on every patient. However, given the space and the location of the incision, there is a maximum number of specialists that could help not only on this part of the procedure, also in the entire surgery. The data collected in this section It was possible to define the following theoretical criteria's.

\& Quiet system.

\& Easy handling.

as No electricity.

is Low cots materials.

a Biocompatible materials.

Those theoretical criteria's are considered the complementary part on the determination of the parameters for the QFD (Figure 2).

\subsection{Surgical requirements.}

Information collected in the surgeries observed and the documentation gave us the parameters considered for this work. The QFD generate the following parameters for the DCR procedure.

1. The RRS should be controlled by the specialist and limited on the pressured supported. 
2. The RRs should be easy handling for the user.

3. Should establish retraction points considering an elliptical geometry.

4. The retraction points should liberate the workspace area and the user's safety.

5. The RSS should consider the different depths needed for the user on the retraction process.

6. The RRS should consider a quiet system for its functioning.

7. RRS should work without electricity.

8. The RRS should be built by low-cost materials.

This analysis needs information not founded on the bibliography, forces, deformations, lengths and the mathematical behaviour of the incision retraction process. In this article the forces, lengths, and deformations resulting in the retraction process. For this part, It is important to generate specifications for the testing protocol.

\subsection{Technical Specifications.}

The system's surgical behaviours are specifications for needed for the RRS construction. Those specifications are listed in Table 2.

Table 2. List of the system requirements.

\begin{tabular}{cl}
\hline \multicolumn{2}{c}{ Requirements } \\
\hline Depth & $2 \mathrm{~mm}$ \\
Length of the major axis & $4 \mathrm{~mm}$ \\
Depth & $10 \mathrm{~mm}$ \\
& $15 \mathrm{~mm}$ \\
\hline
\end{tabular}

\section{Testing protocol.}

This section presents the experimental arraignment for the forces and lengths for a retraction point in the incision. The replication retraction process was performed in a head of a pig. The head of the pig that was chosen with that age and dimensions considering the number of tests to be carried out and the consistency of the skin of an adult pig. Table 3 shows the characteristics of the pig's head.

Table 3. Dimensions, weights, and age of the sample.

\begin{tabular}{cl}
\hline \multicolumn{2}{c}{ Characteristics of the sample. } \\
\hline Part of the animal & Pig head \\
Age & 6 months \\
Weight of the animal & $87 \mathrm{~kg}$ \\
Weight of the head & $1.8 \mathrm{~kg}$ \\
& Length: 480 \\
& Width: 110 \\
Dimensions (mm) & Height: 100 \\
& Yes \\
\hline
\end{tabular}

It is important to mention that the pig head had the Federal Inspection Type (TIF) certification, which is a recognition of quality granted by the SAGARPA, through the National Service of Health, Quality and Agroalimentary Safety (SENASICA).

The testing protocol is divided on the theoretical restrictions that delimit the experimental procedure for the case study and simplify the experimental protocol; Define the retraction points for a homogeneous workspace area; define the number of tests for the skin retraction and the experimental protocol. 


\subsection{Retraction points.}

As the retraction skin process is considered as a mechanical retraction of an elastic material, therefore the plastic strain could be considering a parameterizable study of the forces and the retraction points. However, for a homogeneous workspace area, there should be a minimum of retraction points required. The surgeries observed showed a workspace area similar to an ellipse or with semi-circular behaviour.

The retraction process is attached to the retraction points, but the more points the incision has on the retraction it is more difficult its handling and get less workspace are.

For these reasons the behaviour of the RRS should consider at least six retraction points in an incision. The six points are divided on equidistant locations of the incision and replicates a workspace area of an ellipse.

\subsection{Theoretical restrictions.}

In order to generate the first approach of a mathematical model, restrictions on the system are important. Figure 3 shows 6 retraction points with some restrictions listed below. The variables of the system are time, area, depth, force, and effort.

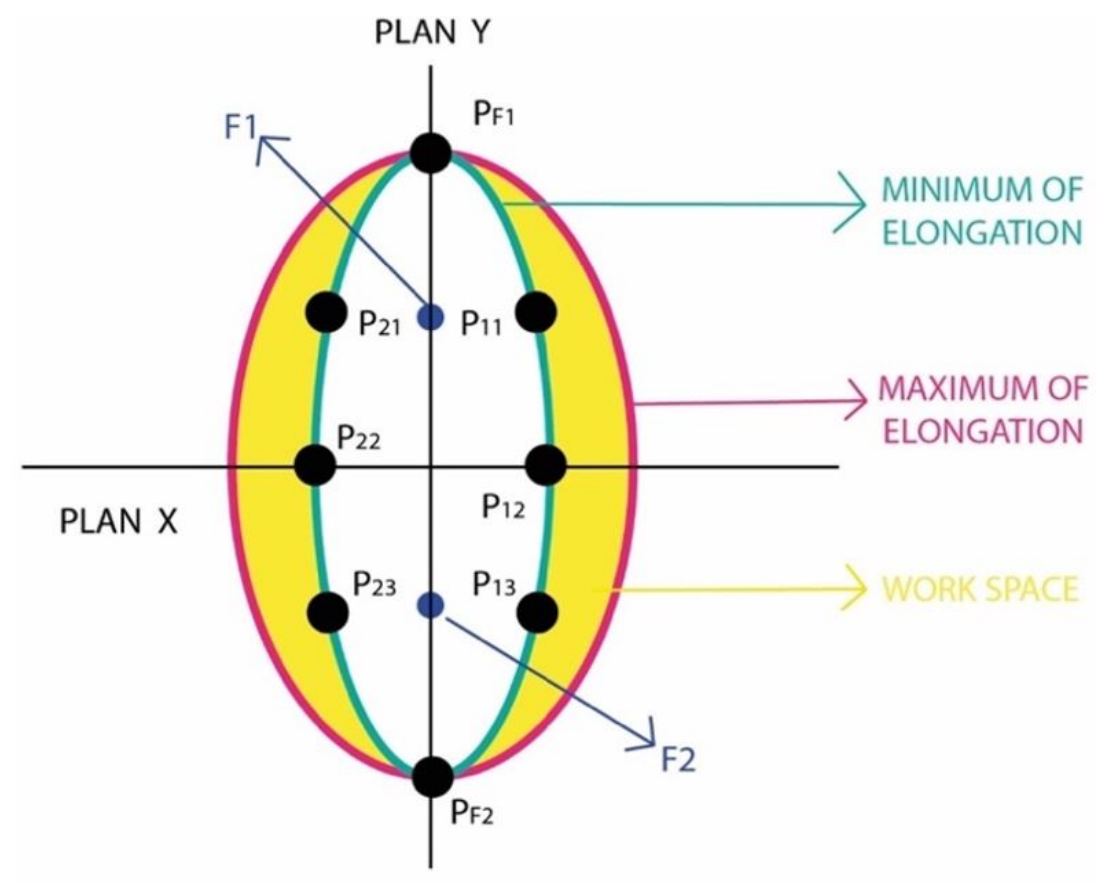

Figure 3. Outline of the theoretical restrictions.

Also, Given the requirements, the system has two depths, 2 and $4 \mathrm{~mm}$. The system variables are time, length and deformations. The depth starts from $0<P<4 \mathrm{~mm}$.

\section{Considerations:}

1. Each incision has a maximum of stretch and a minimum of contraction concerning the origin.

2. The maximum elongations are about the radius of the incision $\left(O P_{F 1}\right)$ or $\left(O P_{F 2}\right)$, which is the radius $(\mathrm{r})$.

3. 3. The points of the incision are coupled to those of an ellip-soid in the following form:

$$
\begin{gathered}
L R=\overline{P_{21} P_{11}}=\overline{P_{23} P_{13} P_{F 1}}=V_{1} \\
P_{F 2}=V_{2} \\
\text { Minoraxis }=\overline{P_{22} P_{12}}
\end{gathered}
$$


4. The opening resembles an ellipse but does not maintain ge-ometry, since it can have an opening that tends to the radius or the middle of the major axis.

$$
\begin{gathered}
0<P_{11}<r \longrightarrow \text { Parallel to the focus } F_{1} \\
0<P_{12}<r \longrightarrow \text { Parallel to the origin } 0 \\
0<P_{13}<r \longrightarrow \text { Parallel to the focus } F_{2} \\
0<P_{21}<r \longrightarrow \text { Paralle to the focus } F_{1} \\
0<P_{22}<r \longrightarrow \text { Parallel to the origin } \\
0<P_{23}<r \longrightarrow \text { Parallel to the focus } F_{2}
\end{gathered}
$$

5. The movements of $\mathrm{P} 11$ and $\mathrm{P} 21, \mathrm{P} 12$ and $\mathrm{P} 22, \mathrm{P} 13$ and $\mathrm{P} 23$ are symmetrical:

$$
\begin{aligned}
& P_{11}(x)=-P_{21}(x) \\
& P_{12}(x)=-P_{22}(x) \\
& P_{13}(x)=-P_{23}(x)
\end{aligned}
$$

6. The independence of the points generates the desired deformation, given that there will be six possible stretching radios.

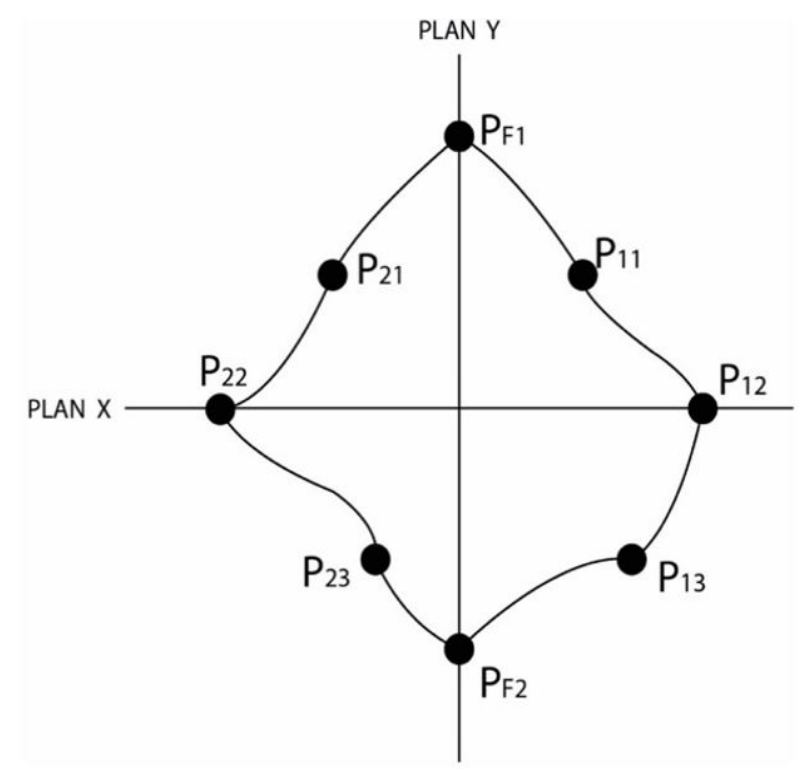

Figure 4. Outline of the theoretical restrictions.

Figure 4 shows real behaviour of the incision during the retraction of the six points necessary to generate a useful workspace.

\subsection{Experimental protocol.}

The experimental protocol is divided into 3 stages, the experimental setup, the testing execution and the data acquisition shown in Figure 5.

For the experimental part, metrology tests were performed on dead tissue with similar characteristics of humans in the area of the eye's obverse muscle, to obtain the parameters of force, area, pressure, and resistance needed to design the RRS in the DCR.

Figure 5 shows the flowchart for the incision retraction on a pig's head. For the acquisition and characterization of the data obtain It was used tool listed in Table 4. 


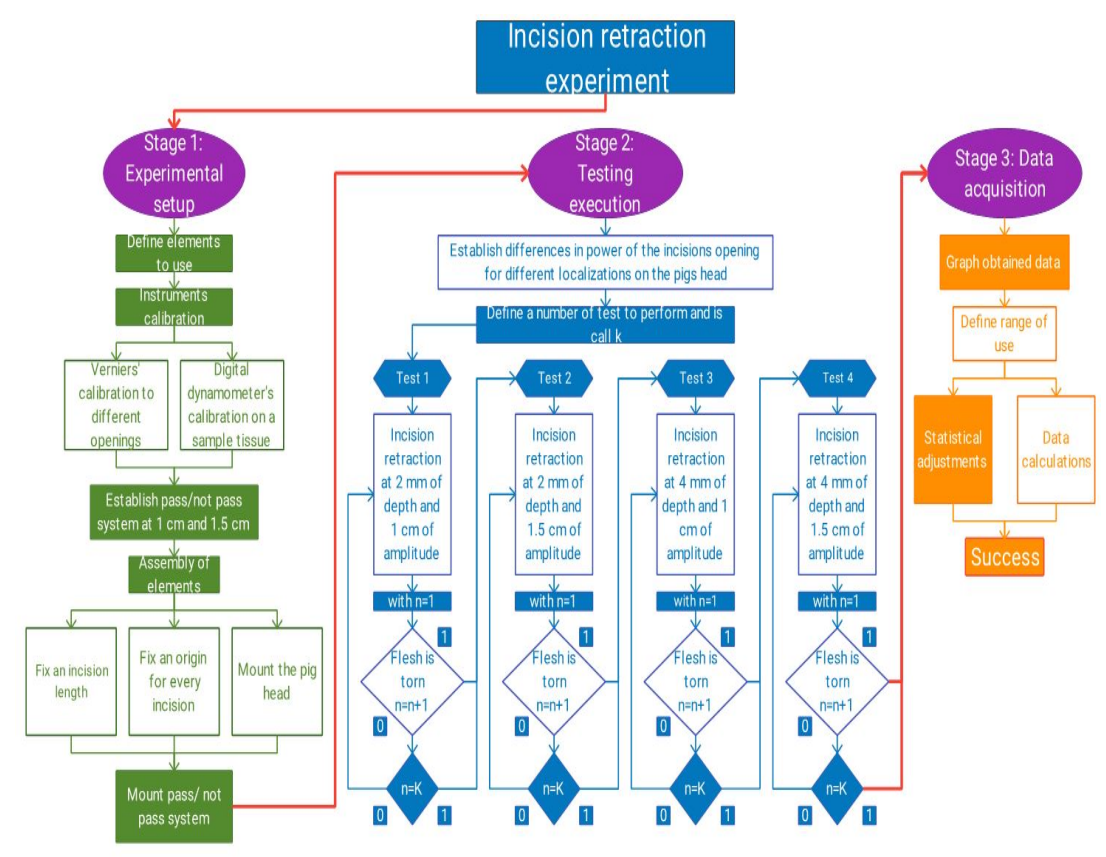

Figure 5. Experimental protocol.

Table 4. Instruments for measurement.

\begin{tabular}{clc}
\hline \hline Instrument & Total & Features \\
\hline $\begin{array}{c}\text { Digital } \\
\text { dynamometer }\end{array}$ & 1 & $\begin{array}{c}\text { PASCO Scientific force } \\
\text { sensor CI-6537 }\end{array}$ \\
\hline Analog Vernier & 2 & $\begin{array}{c}\text { Metromex 333-P plastic } \\
\text { with a range of maximum } \\
\text { measurement of } 5 \mathrm{~mm}\end{array}$ \\
\hline Laser light & 1 & TEKCOPLUS DSTK-110 \\
\hline Universal support & 3 & $\begin{array}{c}\text { Rod of 60 cm and smooth } \\
\text { base of 20 X 14 cm }\end{array}$ \\
\hline \hline
\end{tabular}

\subsection{Procedure.}

With a fixed sample to be measured, the protocol for the measurement of tests was generally carried out, which is composed of three stages that begin in the assembly of the sample, the number of tests to be performed and the acquisition of data. In Figure 1, the flow chart for said protocol is shown.

\section{Experimental results.}

Once the measurements were made, the data was characterized and shown below.

\subsection{Sample space}

Due to the nature of the digital dynamometer, the data obtained cannot be considered in its entirety [22]. The dynamometer starts its measures since the beginning in the skin retraction and finishes the skin is no longer retracted (Figure 6), for this reason, the sample space should consider the retraction forces needed to keep the incision retracted at a defined length. Figure 6 shows the entire measured for the skin retraction. 


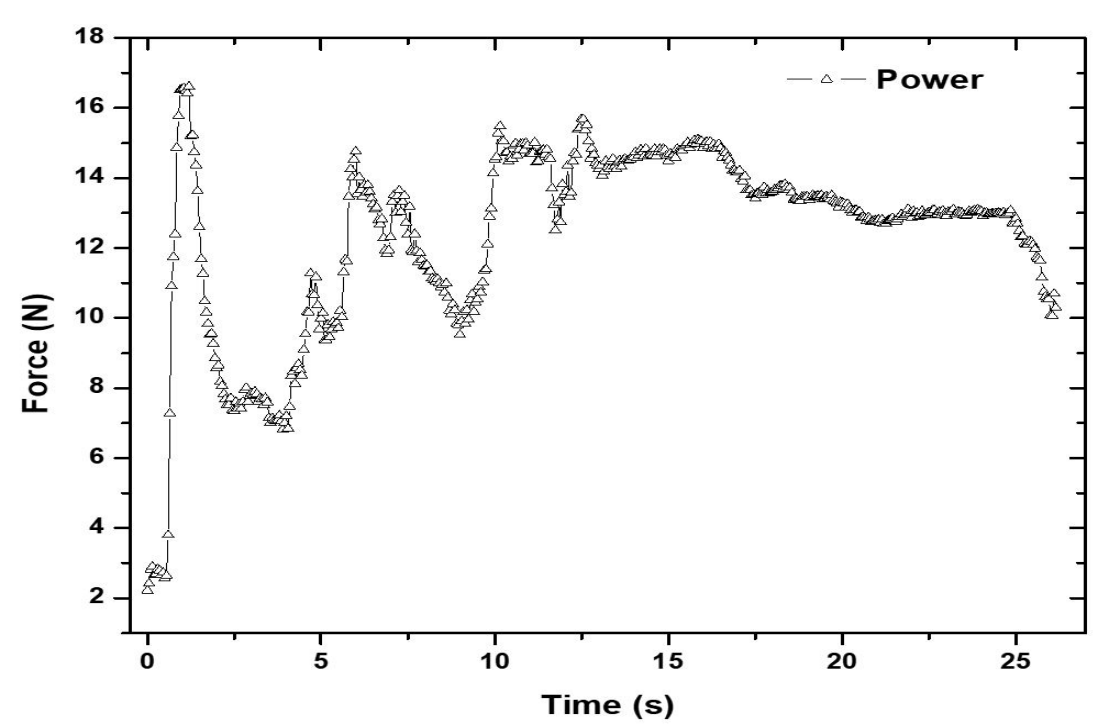

Figure 6. Forces measured during the entire process of opening the incision.

Figure 6 shows the opening process from the start of 0 to 26 seconds. The analysis of the statistical data was defined from 13 to 24 seconds which is the interval where the data kept the incision retracted in the desired length.

\section{Results.}

The data analyzed is related to the power needed to maintain the amplitude required during the experiment, and its analysis will focus on determining the average and maximum force in order to define the force metric in opening incisions in animal tissue.

\subsection{Incision $1 \mathrm{~cm}$ wide and $2 \mathrm{~mm}$ deep.}

Given the experimental data obtained, see Table 5, the non-linear Lorentz adjustment was made to predict its behaviour. This is shown in Figure 5 ??

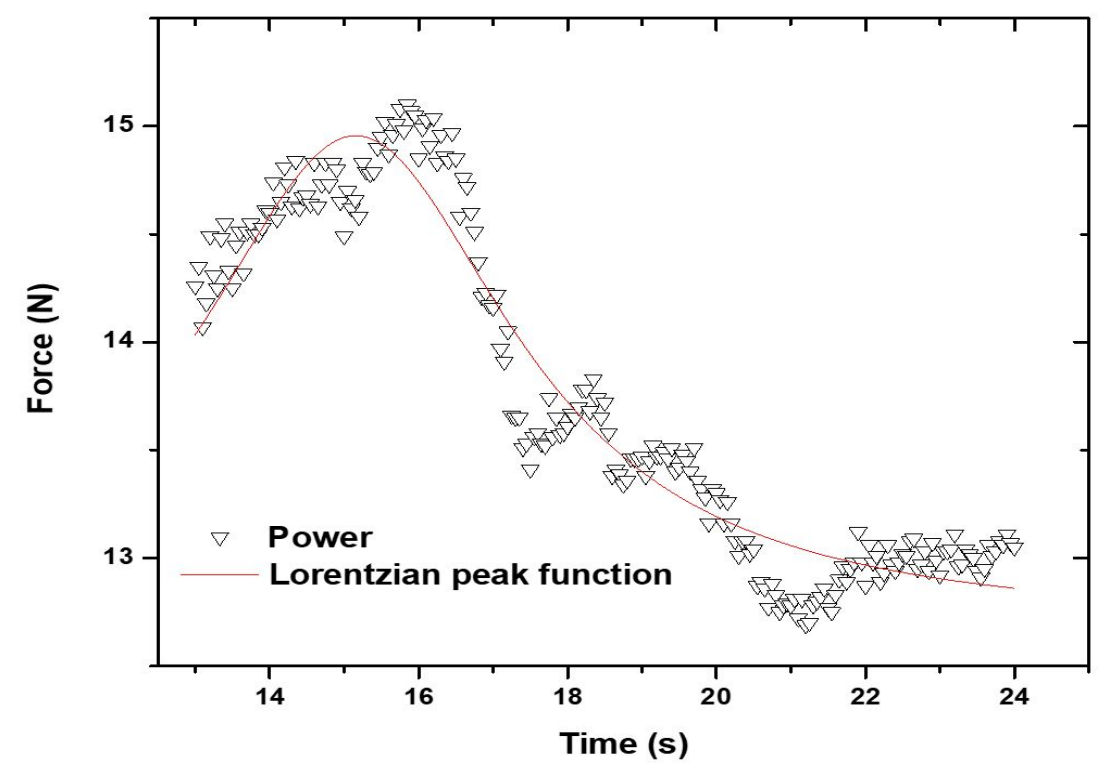

Figure 7. Lorentz adjustment for the experimental test.

Having the following determined average values. Figure 7 shows the behaviour of the retraction in the lapse of 11 seconds. The statistical fit shows how forces in relation with time tends to decrease. 
Table 5. Tests performed at force 1 .

\begin{tabular}{cl}
\hline \multicolumn{2}{c}{ Force test 1} \\
\hline Events & 221 \\
Average & 13.74529 \\
Standard deviation & 0.76547 \\
Variance & 0.58595 \\
Median & 13.52 \\
Maximum & 15.1 \\
Minimum & 12.69 \\
\hline
\end{tabular}

With this adjustment, it is determined that the average force needed to maintain the opening of 1 $\mathrm{cm}$ radius for some time. These data show us that the variation of the media with the maximum value acquired is 1.355 Newtons, and with this, there is a variation between parameters of $9.8 \%$.

\subsection{Incision $1 \mathrm{~cm}$ wide and $4 \mathrm{~mm}$ deep.}

Given the experimental data obtained, the non-linear Lorentz adjustment was made to predict its behaviour. This is shown in Figure 8. Having the following determined average values.

Table 6. Tests performed at force 2 .

\begin{tabular}{cl}
\hline \multicolumn{2}{c}{ Force test 2} \\
\hline Events & 221 \\
Average & 18.88036 \\
Standard deviation & 0.96741 \\
Variance & 0.93588 \\
Median & 18.81 \\
Maximum & 20.91 \\
Minimum & 16.4 \\
\hline
\end{tabular}

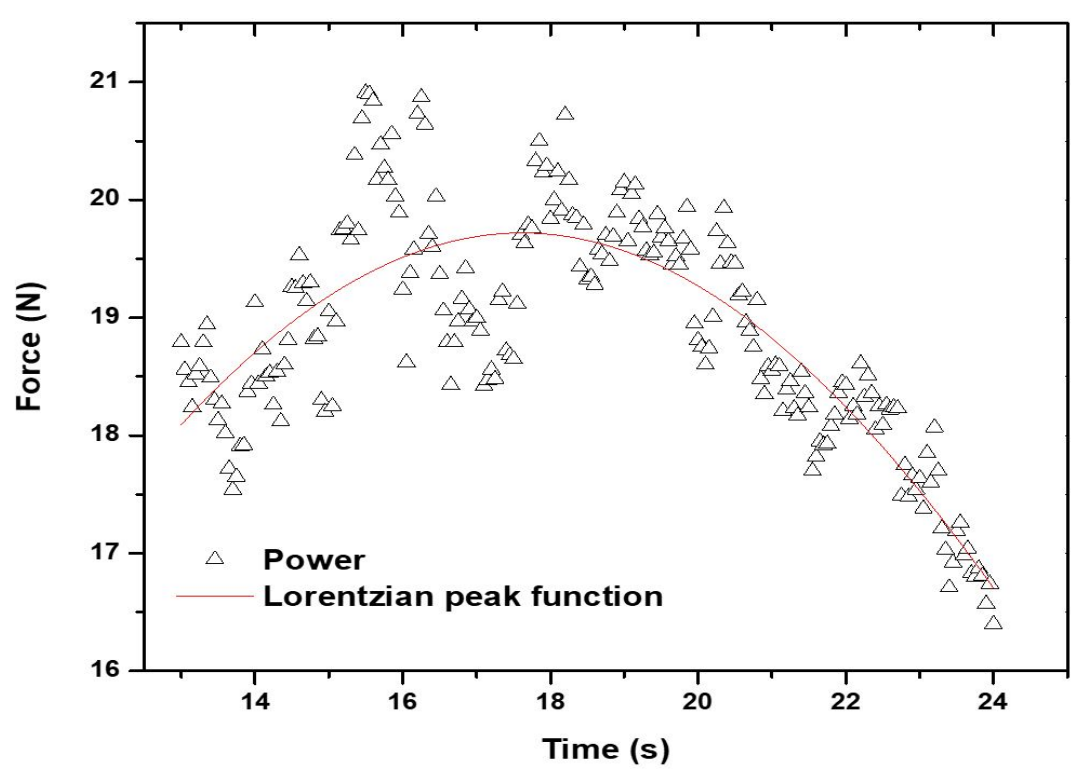

Figure 8. Lorentz adjustment for the experimental test.

With this adjustment, it is determined that the average force needed to maintain the opening of 1 $\mathrm{cm}$ radius for some time. These data show us that the variation of the mean with the maximum value acquired is 2.03 Newtons, and with this, we have a variation between parameters of $10.7 \%$. 


\subsection{Incision $1.5 \mathrm{~cm}$ wide and $4 \mathrm{~mm}$ deep.}

Given the experimental data obtained, the non-linear Lorentz adjustment was made to predict its behaviour. This is shown in Figure 9. Having the following determined average values.

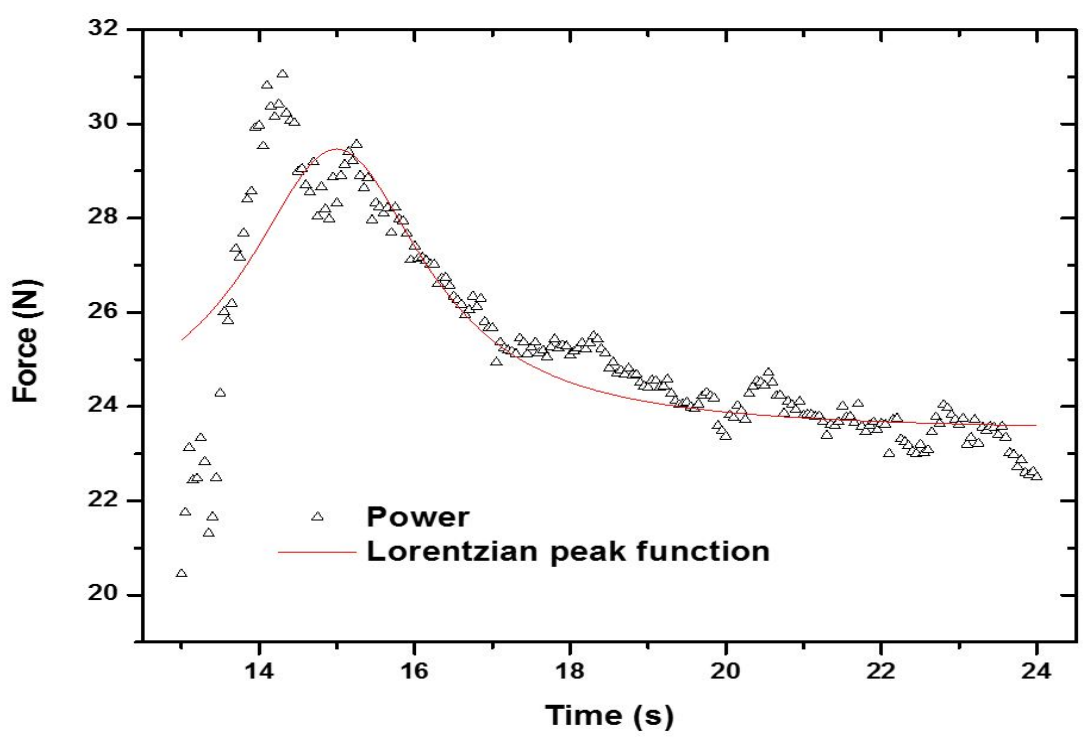

Figure 9. Lorentz adjustment for the experimental test.

Table 7. Tests performed at force 3.

\begin{tabular}{cl}
\hline \multicolumn{2}{c}{ Force test 3 } \\
\hline Events & 221 \\
Average & 25.26041 \\
Standard deviation & 2.17057 \\
Variance & 4.71137 \\
Median & 24.55 \\
Maximum & 31.05 \\
Minimum & 20.45 \\
\hline
\end{tabular}

With this adjustment, it is determined that the average force necessary to maintain the opening of $1.5 \mathrm{~cm}$ radius during a period. These data show us that the variation of the mean with the maximum value acquired is 5.79 Newtons, and with this, we have a variation between parameters of $20 \%$.

\section{Analysis of the average curve.}

Having the three forces, a point-to-point average of the measured forces was generated during the same interval. These averages are shown in Figure 10.

Table 8. Tests performed for all the forces

\begin{tabular}{cl}
\hline \multicolumn{2}{c}{ Three forces average test } \\
\hline Events & 221 \\
Average & 18.88036 \\
Standard deviation & 0.96741 \\
Variance & 0.93588 \\
Median & 18.81 \\
Maximum & 20.91 \\
Minimum & 16.4 \\
\hline
\end{tabular}




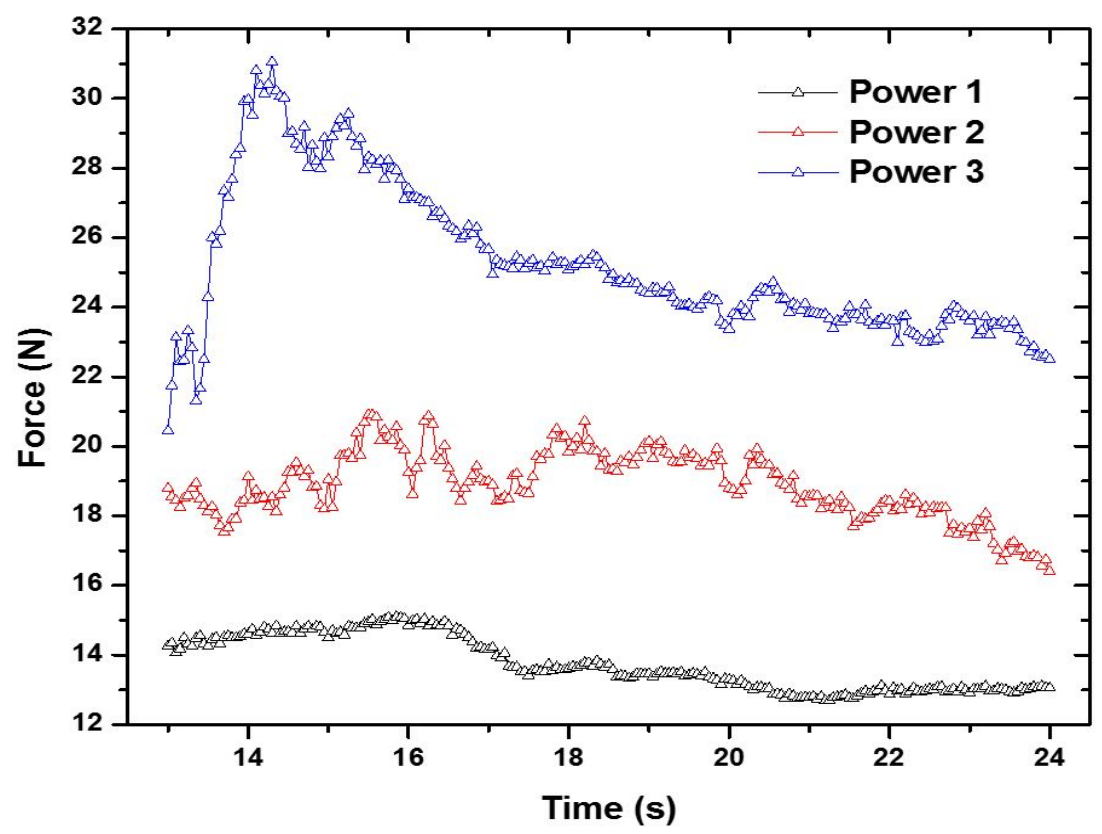

Figure 10. Lorentz adjustment for the experimental test for the aver-age of all the forces.

Finally, with the forces and lengths measured. It could be possible to determine the areas retracted on the experiment. These areas are based on the mathematical model which relates areas and forces on Mohr circles theory. The areas and lengths areas are shown in Table 9.

Table 9. Areas and lengths for the forces measured.

\begin{tabular}{ccccc}
\hline \multicolumn{4}{c}{ Areas and lengths } \\
\hline \multicolumn{2}{c}{ Radii of the Mohr circles(m) } & \multicolumn{2}{c}{ Areas $\left(m^{2}\right)$} \\
Radius 1 & Radius 2 & Radius 3 & Area 1 & Area 2 \\
0.01 & 0.015 & 0.02 & 0.00001 & 0.00002 \\
\hline
\end{tabular}

With the areas and forces, it is possible to calculate the de-formations for the pigskin in the head. Those deformations are shown in Table 10. The efforts could determine how the incisions behave during the retraction of the skin.

Table 10. Areas and lengths for the forces measured.

\begin{tabular}{cccccc}
\hline \multicolumn{5}{c}{ Mohr Circles } \\
\hline \multirow{3}{*}{ Forces (N) } & & \multicolumn{3}{c}{ Sigma (F/A) } \\
13.74 & 18.88 & 25.26 & 1374000 & 944000 & 1263000 \\
\hline
\end{tabular}

As the data analyzed are set at the same time, the independent data involved could show the behaviour of the retraction during its maximal length of retraction regardless of the different depths. This is exemplified in Figure 11.

Figure 11 shows the minimal and maximal tolerances that a retractor system could have in order to keep open an incision on a surgical procedure. 


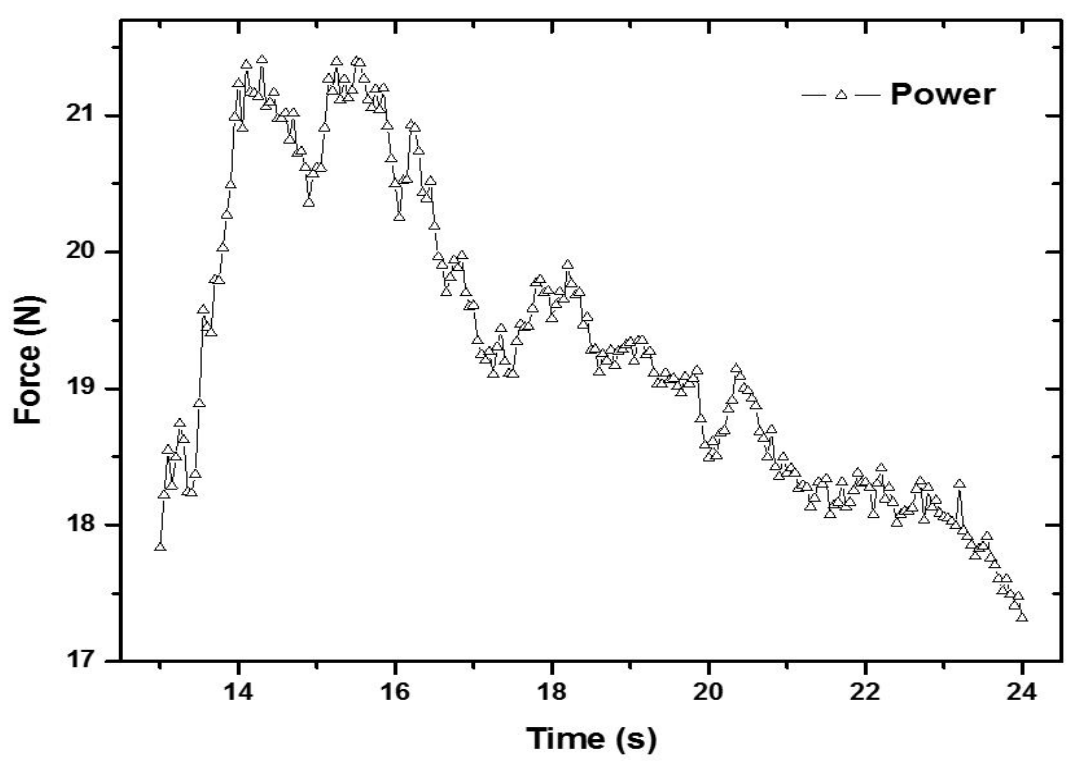

Figure 11. Graph of media behaviour for the three powers measured.

\section{Conclusions}

The effects inflicted by the retraction forces are related on the type of incision, the retractions points, the zone where the incision was made and the time in which the incision was retracted; Those factors alter the deformation and behaviour of the skin.

Figure 6 can be inferred that the behaviour of the skin tends to be stable with the time, and the forces needed to keep the working area open decreases with the time. This behaviour suggests that the RRS should have tolerances within the variance of each type of incision.

The skin retraction was performed for a point the incision, but due to the characteristics of the skin and the metrology treatment of the data measured it could be inferred that the data obtained could be scalable on forces and lengths. Figure 10 shows the average graph of the three points measured and shows the same behaviour as each incision.

The retraction process does not achieve a homogeneous workspace area and in order to generate the desired workspace area, it is suggested to use at least six retraction points, three for each side of the incision. These six points could open the area of the incision similar to an ellipse which is considered the ideal design in the construction of a RRS auxiliary on DCR.

The total experimental data measured for each test cannot be used, this is due to the fact that these are related to the time and their beginning of measurement included from the zero point of retraction, passing through keeping the open incision the required area, until the relaxation of the tissue where the retraction is finished (Figure 4). The experimental data should be considered in a defined period and, for this work, the period in which the opening of the incision was contemplated from 13 to 24 seconds.

The experimental data are considering that the material (the skin) is used as a flexible element since at the point that the material is torn, the practice stopped. Therefore, the experimental data shown are exclusively for elastic materials on the ocular cavity on procedures such as DCR.

The Lorentz distribution was defined as the adjustment to be used because its mode and median are well defined, as well as for the use for the analysis of independent and identically distributed events.

For the test realized shows a variation of parameters between the three forces measured of 5.315 Newtons, which represents the $15 \%$ of the variation between forces needed to open the incision. This number could represent tolerances for an actuator in order to keep a workspace. 


\section{Patents}

The presented mathematical model generates an device patented in the Mexican Institute of Industrial Property (IMPI) named Patent of a calibrator device of ocular surgery retraction and it has the registration number MX/F/2019/003098.

Author Contributions: V.O.R.B. developed the practical aspects of this research, created the mathematical model and wrote the first draft of this paper, L.A.Z.A provided the initial formulation of the methodology and do exhaustive work on reviewing and editing, R.M.V.R, J.J.R.L. and G.M.C.M supervised this research. All authors participated in reviewing and writing of this manuscript.

Funding: This research was funded by CONACYT (Consejo Nacional de Ciencia y Tecnología) grant number 863564 .

Acknowledgments: The authors would like to thank Surgeon Ángel Nava Castañeda for all the support during the the lacrimal surgeries. Authors also would like to thank al the patients help, effort during the research study, the metrology lab staff at the UAEM for their assistance in the experiment period. The authors specially would like to thank Ophthalmologist Luz Maria Mendez for all the assistance, tolerance and for wrote the user satisfaction letter with official date on October 10th 2019. We acknowledge the support from Engineers and medics for providing the testing bench used in this research and for allowing us to conduct the experimental analysis at their labs. Likewise, we acknowledge the support of the Mexican Council for Science and Technology (CONACYT) and the Autonomous University of Mexico State for funding this research.

Conflicts of Interest: The authors declare that they have no conflict of interest.

\section{References}

1. Staff, I.M. Las personas con discapacidad en México: una visión censal; Instituto Nacional de Estadística, Geografía e Informática, 2004.

2. de Estadística (Honduras), I.N.; de Estado en el Despacho Presidencial, H.S. XVI Censo de Población y V de Vivienda: Características económicas de la población interrelacionadas; Vol. 5, Instituto Nacional de Estadística, 2004.

3. Arranz-Márquez, E.; García-González, M.; Teus, M. Disminución de la agudeza visual. Medicine-Programa de Formación Médica Continuada Acreditado 2015, 11, 5423-5432.

4. Root, T. OphthoBook; Royal New Zealand Foundation of the Blind, 2011.

5. Kamal, S.; Ali, M.; Gauba, V.; Qasem, Q. Principles and Practice of Lacrimal Surgery 2015.

6. Presutti, L.; Mattioli, F. Endoscopic Surgery of the Lacrimal Drainage System; Springer, 2016.

7. Walland, M.J.; Rose, G.E. Soft tissue infections after open lacrimal surgery. Ophthalmology 1994, 101, 608-611.

8. Piermattei, D.; Johnson, K. An Atlas of Surgical Approaches to the Bones and Joints of the Dog and Cat Saunders, 2004.

9. Schmook, F.P.; Meingassner, J.G.; Billich, A. Comparison of human skin or epidermis models with human and animal skin in in-vitro percutaneous absorption. International journal of pharmaceutics 2001, 215, 51-56.

10. Netzlaff, F.; Schaefer, U.F.; Lehr, C.M.; Meiers, P.; Stahl, J.; Kietzmann, M.; Niedorf, F. Comparison of bovine udder skin with human and porcine skin in percutaneous permeation experiments. Alternatives to laboratory animals: ATLA 2006, 34, 499-513.

11. Burger, J.; Van't Riet, M.; Jeekel, J. Abdominal incisions: techniques and postoperative complications. Scandinavian Journal of Surgery 2002, 91, 315-321.

12. Mathai, M.; Hofmeyr, G.J.; Mathai, N.E. Abdominal surgical incisions for caesarean section. Cochrane Database of Systematic Reviews 2013.

13. Hu, L.W.; Paul, D.J.; Reis, E.E.; Green, I.H.L. Surgical retractor apparatus for operating on the heart through an incision, 2001. US Patent 6,331,158.

14. Weber, R.K.; Keerl, R.; Schaefer, S.D.; Rocca, R.C. Atlas of lacrimal surgery; Springer Science \& Business Media, 2007.

15. Kazama, S. Heart retractor, 1996. US Patent 5,509,890.

16. García, T. Técnicas conversacionales para la recogida de datos en investigación cualitativa: La entrevista (I). Nure investigación 2008, 33.

17. Glick, P. How reliable are surveys of client satisfaction with healthcare services? Evidence from matched facility and household data in Madagascar. Social science \& medicine 2009, 68, 368-379. 
18. Arteaga, O. Investigación en salud y métodos cualitativos. Cienc. Trab 2006, pp. 151-153.

19. Cullen, R. Perspectives on user satisfaction surveys 2001.

20. Jolly, R.; Sanderson, D. A Mohr circle construction for the opening of a pre-existing fracture. Journal of Structural Geology 1997, 19, 887-892.

21. Joodaki, H.; Panzer, M.B. Skin mechanical properties and modeling: A review. Proceedings of the Institution of Mechanical Engineers, Part H: Journal of Engineering in Medicine 2018, 232, 323-343.

22. Rohatgi, V.K.; Saleh, A.M.E. An introduction to probability and statistics; John Wiley \& Sons, 2015. 